\title{
Symptoms of chronic rhinosinusitis differentially impact general health-related quality of life*
}

\author{
Lloyd P. Hoehle1,2, Katie M. Phillips, ${ }^{1,2}$, Regan W. Bergmark',2, \\ David S. Caradonna ${ }^{2,3}$, Stacey T. Gray ${ }^{1,2}$, Ahmad R. Sedaghatt,2,3, \\ 'Department of Otolaryngology, Massachusetts Eye and Ear Infirmary, Boston, MA, USA \\ Department of Otolaryngology, Harvard Medical School, Boston, MA, USA \\ Division of Otolaryngology, Beth Israel Deaconess Medical Center, Boston, MA, USA \\ ${ }^{4}$ Department of Otolaryngology and Communications Enhancement, Boston Children's Hospital, Boston, MA, USA
}

Rhinology 54: 316-322, 2016

DOI:10.4193/Rhino16.211

*Received for publication:

June 7, 2016

Accepted: July 22, 2016

\begin{abstract}
Background: The degree to which different sinonasal symptoms contribute to the overall quality of life (QOL) detriment in chronic rhinosinusitis (CRS) patients remains unknown. In this study we sought to characterize the effect of different CRS symptoms on the general health-related QOL in patients.

Methodology: We performed a prospective cross-sectional study of 131 adult patients with CRS. Sinonasal symptoms were evaluated using the 22-item Sinonasal Outcomes Test (SNOT-22) and general health-related QOL was evaluated using the EuroQol 5-Dimensional general health-related QOL survey (EQ5D) and visual analog scale (EQ5D-VAS). Health utility values (HUV) were determined using responses to the EQ5D. SNOT-22 scores were broken down into subdomain scores for sleep, nasal, otologic/facial pain and emotional function symptoms.
\end{abstract}

Results: The otologic/facial pain subdomain score consistently had the largest impact on EQ5D-VAS and HUV. After otologic/facial pain, the sleep subdomain score had the second largest effect while the nasal subdomain score had the least impact on general health-related QOL.

Conclusions: Different types of CRS symptoms - most prominently otologic/facial pain and sleep-related symptoms-and their underlying pathophysiologic mechanisms may differentially affect the general health-related QOL detriment associated with CRS. These findings raise the possibility that treatment of the various symptoms associated with CRS may lead to differential improvement in general-health related QOL.

Key words: chronic rhinosinusitis, quality of life, sinonasal symptoms, SNOT-22, EQ5D

\section{Introduction}

Chronic rhinosinusitis (CRS), characterized by chronic inflammation of the sinonasal mucosa, leads to sinonasal symptomatology such as congestion, mucopurulent drainage, facial pain/pressure and hyposmia ${ }^{(1,2)}$. From the standpoint of pathophysiology, CRS is a multifactorial process without one specific underlying etiologic process ${ }^{(3,4)}$. Derangements in innate immunity, the sinonasal epithelial barrier, and response to bacterial flora as well as allergic inflammation and sinonasal obstruction have all been implicated in the pathogenesis of $\mathrm{CRS}^{(5-9)}$. The complexity of CRS is further highlighted by the frequent lack of correlation between sinonasal symptomatology and objective findings, such as radiographic disease severity ${ }^{(10,11)}$.
Regardless of etiology, the goal of treatment of CRS is to reduce sinonasal symptomatology. CRS sinonasal symptomatology has previously been shown to have a significant negative impact on patient quality of life (QOL) that is similar to or worse than other chronic diseases, such as asthma or cardiac disease ${ }^{(12)}$. Care of patients with CRS results in billions of dollars in cost every year (13). This expense includes direct healthcare costs that arise from physician visits, medical and surgical treatment, as well as indirect costs that arise from lost productivity.

Various validated measures exist to quantify patient QOL; some metrics, such as the 22-item Sinonasal Outcomes Test (SNOT22), are specific to CRS while others measure general QOL and are applicable to many different conditions ${ }^{(14)}$. Measures of 
general health-related QOL are particularly important because they can be standardized across multiple conditions for direct comparisons of disease burden and they can also be used to study the cost-effectiveness of various interventions ${ }^{(15,16)}$. With these validated tools, treatment of CRS, including endoscopic sinus surgery (ESS), has been shown to improve general healthrelated QOL, demonstrating the cost-effectiveness and utility of various treatment modalities ${ }^{(17-19)}$.

While the symptoms of CRS overall are well known to be associated with general health-related QOL impairment, it remains unclear whether particular symptoms contribute more than others ${ }^{(20)}$. Previous work has shown that sleep, nasal, otologic/ facial pain, or emotional function symptoms may be the most dominant symptoms in subsets of CRS patients ${ }^{(21,22)}$. In this present study, we seek to determine whether these subsets of CRS symptomatology are differentially associated with the general health-related QOL deficit associated with CRS.

\section{Materials and methods}

\section{Study participants}

This study was approved by the Massachusetts Eye and Ear Infirmary Human Studies Committee. A total of 131 adult patients (with age 18 years or older) with CRS were recruited prospectively and provided informed consent for inclusion in this study. All participants met consensus guideline established criteria for $\mathrm{CRS}^{(1,2)}$. Exclusion criteria included comorbid diagnoses of: 1) vasculitis, 2) cystic fibrosis, 3) sarcoidosis, and 4) immunodeficiency. Any patient having undergone ESS within 6 months was also excluded.

\section{Study design and data collection}

This investigation is a prospective cross-sectional study of the impact of CRS symptomatology on general health-related QOL. All data were collected at enrollment. The age and gender of all participants were recorded and all participants completed the validated SNOT-22 ${ }^{(23)}$, which reflects sinonasal symptomatology (and CRS-specific QOL), and the EuroQol 5-dimensional (EQ5D) general health survey and visual analog scale (EQ5D-VAS)(15), which reflects general health-related QOL. Health utility values were derived, as previously described ${ }^{(15)}$, from the responses to the EQ5D. At enrollment, participants were assessed by the evaluating rhinologist for a history of 1) aeroallergen hypersensitivity based on formal testing, 2) asthma, and 3) nasal polyps.

Classification and quantification of sinonasal symptoms into subdomain scores based on SNOT-22 responses

We classified sinonasal symptoms in four categories: sleep, nasal, otologic/facial pain and emotional function symptoms. For each category, we used two methods to derive corresponding subdomain scores using the SNOT-22. In the first method, we used principal component analysis (PCA). Previous work has shown that the first four principal components (PCs) from PCA performed on SNOT-22 responses of CRS patients are dominated by sleep, nasal, otologic/facial pain and emotional function symptoms, respectively ${ }^{(22)}$. Upon confirming this to be the case for our study participants by performing a PCA on their SNOT22 responses, we calculated a PC-derived SNOT-22 subdomain score for each of these symptom categories by weighting the normalized score for each SNOT-22 item by the loadings on the corresponding PC, as previously described ${ }^{(22)}$. In the second method, we calculated an un-weighted SNOT-22 subdomain score for each symptom category. Based on the dominant symptoms that we found in each PC, un-weighted SNOT-22 subdomain scores were calculated by simply adding the responses to questions \#11 through \#18 for the un-weighted sleep symptoms subdomain score; questions \#1 through \#6, \#21 and \#22 for the un-weighted nasal symptoms subdomain score; questions \#7 through \#10 for the un-weighted otologic/facial pain subdomain score; and questions \#19 and \#20 for the un-weighted emotional function subdomain score.

\section{Statistical analysis}

All analysis was performed with the statistical software package R (www.r-project.org). Correlations were assessed using Pearson's method. A total of 131 participants were recruited to power this study to detect a statistically significant association of medium effect $(r=0.15)^{(24)}$ in a multivariable linear regression between EQ5D-VAS and nine independent variables (any one of our four symptom subdomains and up to five additional covariates) at a significance level of 0.05 with power of 0.90 using the pwr package. PCA was performed on the SNOT-22 responses of all study participants. Determination of variable loading onto each PC was determined with a varimax rotation on the original PCA using the principal function of the psych package. The four PCs, which explained the greatest amount of data variability, were identified as the first four PCs. Univariate and multivariable associations between PC-derived composite scores and subdomain scores (as independent variables) and EQ5D-VAS and HUV (as dependent variables) were calculated using linear regression models.

\section{Results}

\section{Study participants}

A total of 131 participants (53.4\% male and $46.6 \%$ female) were enrolled with mean age of 51.4 (standard deviation [SD]: 15.4). Of these participants, $49.6 \%$ had a history of aeroallergen hypersensitivity, $29.8 \%$ had a diagnosis of asthma, $46.6 \% \mathrm{had}$ nasal polyps and $33.6 \%$ had a history of prior endoscopic sinus surgery for CRS. The mean SNOT-22 score was 36.2 (SD: 23.1), the mean EQ5D-VAS was 73.7 (SD: 19.2) and the mean HUV was 0.86 (SD: 0.14).

PCA performed on the SNOT-22 responses revealed that the first 


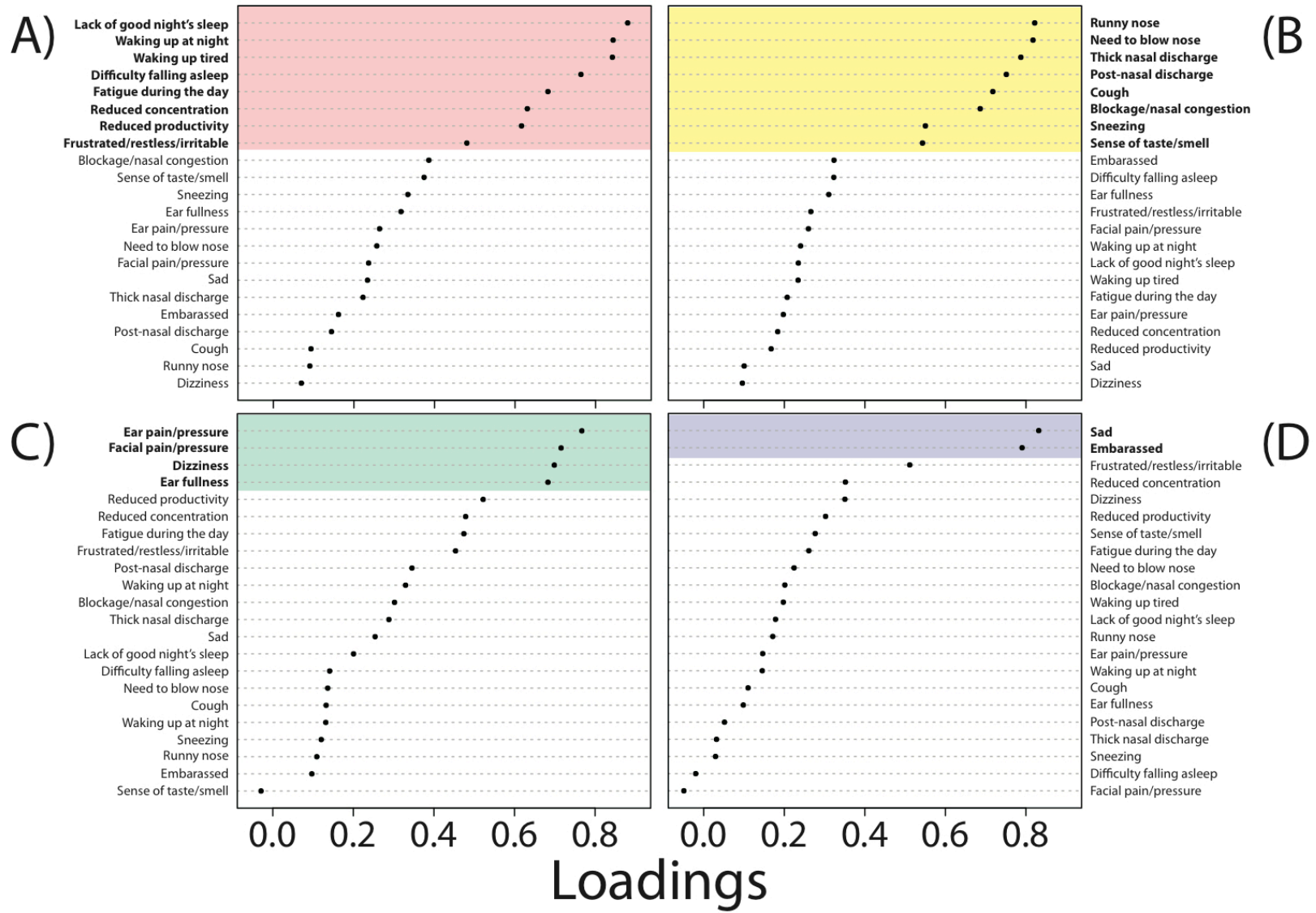

Figure 1. Loadings for each SNOT-22 response item for (A) the first principal component that is dominated by sleep symptoms, (B) the second principal component that is dominated by nasal symptoms, (C) the third principal component that is dominated by otologic/facial pain symptoms, and (D) fourth principal component that is dominated by emotional function symptoms.

four PCs were dominated by sleep, nasal, otologic/facial pain and emotional function symptoms, respectively (Figure 1), from which PC-derived subdomain scores were calculated. Un-weighted SNOT-22 subdomain scores for sleep, nasal, otologic/facial pain and emotional function symptoms were likewise calculated with mean of 13.9 (SD: 10.8) for the un-weighted sleep subdomain score, 16.1 (SD: 9.7) for the un-weighted nasal subdomain score, 5.0 (SD: 4.7) for the un-weighted otologic/facial pain subdomain score and 1.3 (SD: 2.1) for un-weighted emotional function subdomain score.

CRS symptom subtypes are differentially associated with general health-related QOL impairment

The total SNOT-22 score was significantly correlated with the EQ5D-VAS ( $\rho=-0.53,95 \% \mathrm{Cl}:-0.64--0.39, \mathrm{p}<0.001)$ and the EQ5D-derived HUV ( $\rho=-0.53,95 \% \mathrm{Cl}:-0.64--0.40, p<0.001)$. We next checked whether PC-derived sleep, nasal, otologic/facial pain and emotional function subdomain scores were associated with EQ5D-VAS (Figure 2) and HUV (Figure 3). We found that all PC-derived subdomain scores were significantly associated with EQ5D-VAS while only the sleep, otologic/facial pain, and emotional function PC-derived subdomain scores were significantly associated with HUV by univariate association (Table 1). After multivariable analysis controlling for all PC-derived SNOT-22 subdomain scores as well as age, gender, aeroallergen hypersensitivity, asthma and nasal polyps, all four PC-derived SNOT-22 subdomain scores exhibited a statistically significant association with both EQ5D-VAS and HUV (Table 1). The PC-derived otologic/facial pain subdomain score had the greatest impact on EQ5D-VAS ( $\beta=-6.49,95 \% \mathrm{Cl}:-9.47--3.51, \mathrm{p}<0.001)$ and on HUV $(\beta=-0.06,95 \% \mathrm{Cl}:-0.08--0.03, p<0.001)$. The PC-derived sleep subdomain score had the second largest effect on QOL and the PC-derived nasal subdomain score had the smallest effect on QOL (Table 1).

We similarly checked un-weighted sleep, nasal, otologic/facial pain and emotional function SNOT-22 subdomain scores for association with EQ5D-VAS (Figure 4) and HUV (Figure 5). All of these SNOT-22 subdomain scores were significantly associated with EQ5D-VAS and HUV by univariate analysis (Table 2). However, after multivariable analysis, only the un-weighted sleep subdomain score significantly associated with EQ5D-VAS ( $\beta=$ $-0.55,95 \% \mathrm{Cl}:-0.96--0.13, \mathrm{p}=0.011)$ and only the un-weighted otologic/facial pain subdomain score was significantly associated with HUV ( $\beta=-0.01,95 \% \mathrm{Cl}:-0.02--0.00, p=0.004)$. 


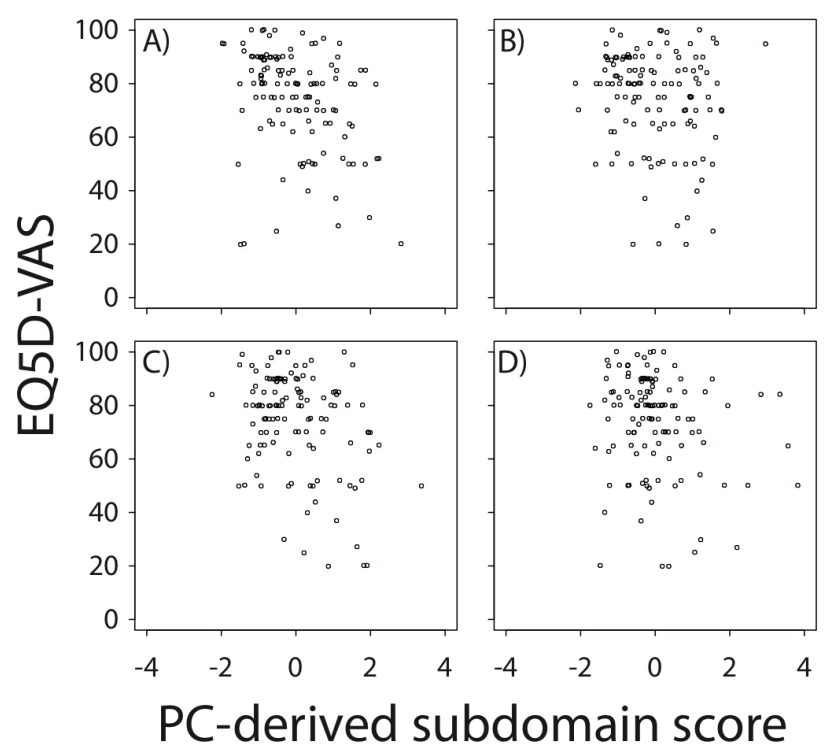

Figure 2. Plots of EQ5D-VAS vs. principal component (PC)-derived SNOT22 subdomain scores for the (A) the sleep subdomain, (B) nasal subdomain, (C) otologic/facial pain subdomain, and (D) emotional function subdomain.
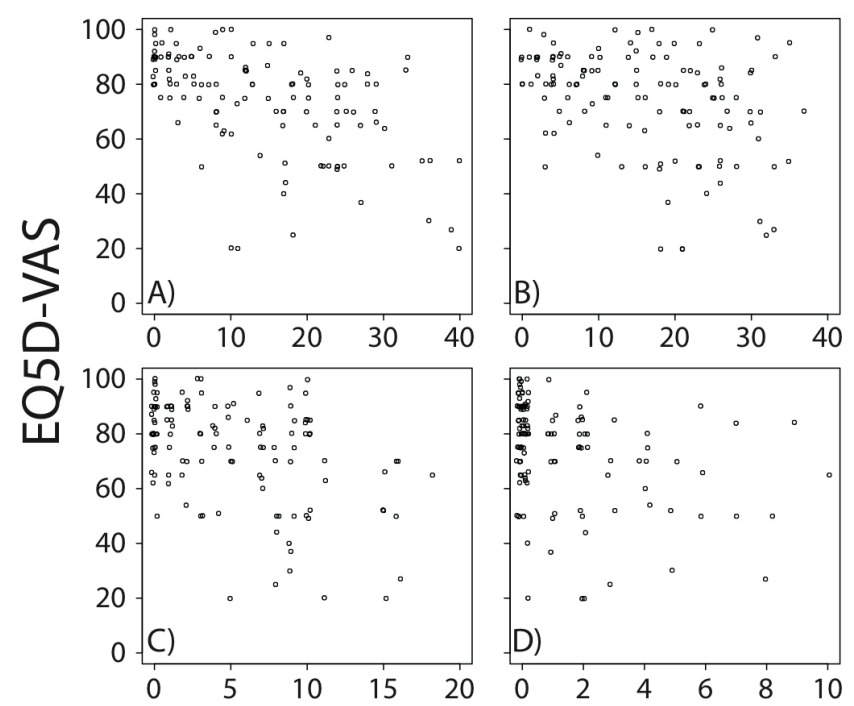

Un-weighted subdomain score

Figure 4. Plots of EQ5D-VAS vs. un-weighted SNOT-22 subdomain scores for the (A) the sleep subdomain, (B) nasal subdomain, (C) otologic/facial pain subdomain, and (D) emotional function subdomain.

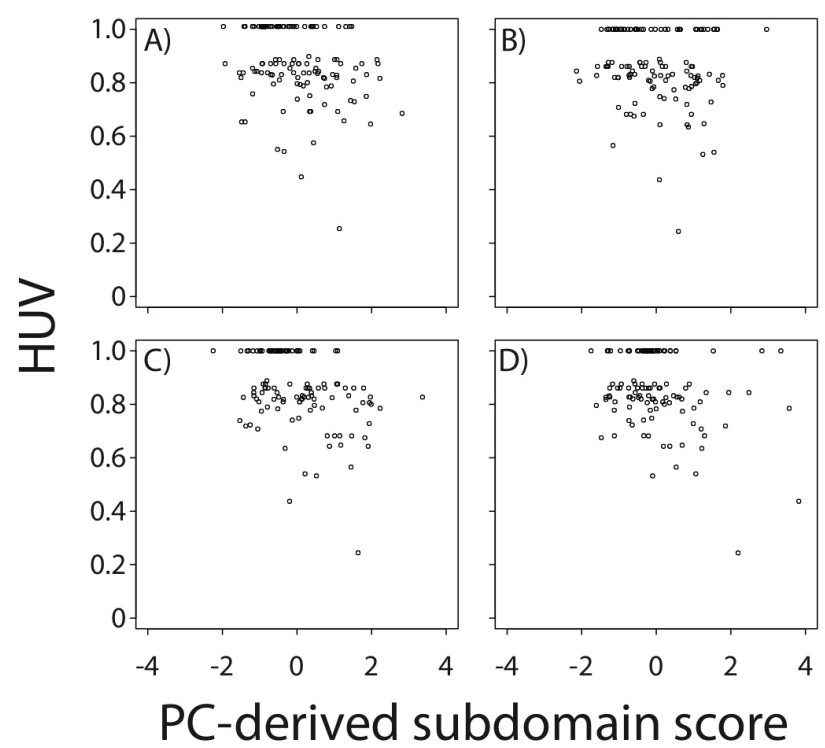

Figure 3. Plots of HUV vs. principal component (PC)-derived SNOT-22 subdomain scores for the (A) the sleep subdomain, (B) nasal subdomain, (C) otologic/facial pain subdomain, and (D) emotional function subdomain.

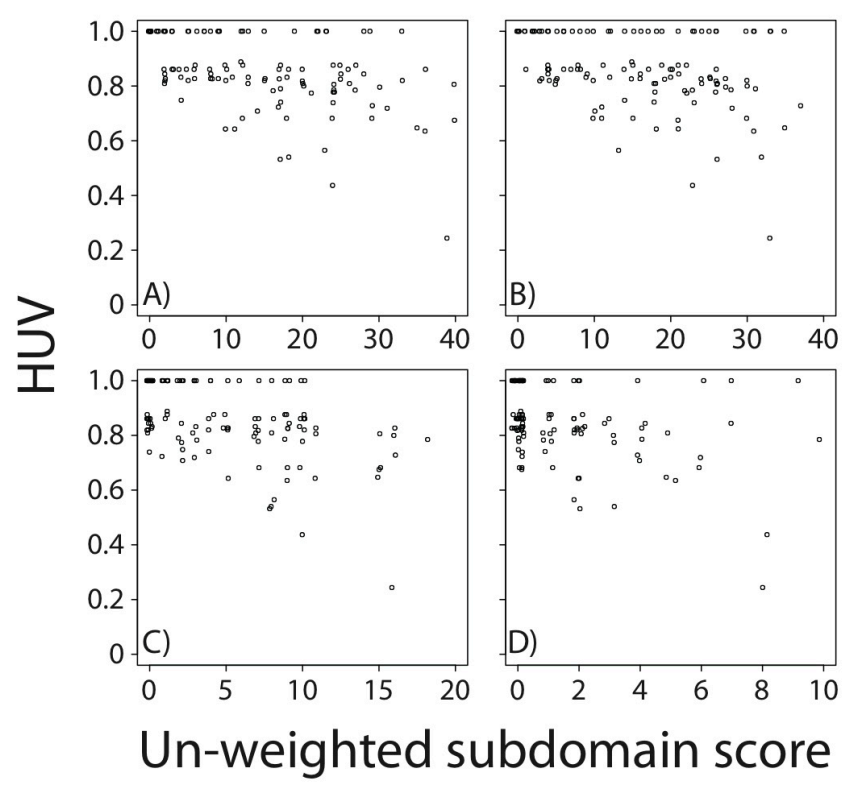

Figure 5. Plots of HUV vs. un-weighted SNOT-22 subdomain scores for the (A) the sleep subdomain, (B) nasal subdomain, (C) otologic/facial pain subdomain, and (D) emotional function subdomain.

\section{Discussion}

CRS causes a significant impairment in QOL that leads to lost productivity at home and at work, which translates to billions of dollars in cost every year ${ }^{(2,12)}$. Many studies have shown that the severity of CRS-specific symptomatology, taken as a whole, is associated with diminished general health-related $\mathrm{QOL}^{(25)}$. However, no study has examined whether specific CRS symptoms may differentially contribute to this general health-related QOL impairment. In this study, we now show that the pathophysiology of CRS relating to otologic/facial pain and sleep symptomatology most contribute to general health-related QOL impairment while nasal symptoms contribute the least to general healthrelated QOL impairment.

Investigating the impact of specific symptomatology may be particularly important because previous work has shown that the different CRS symptom types are associated with specific 
Table 1. Association between principal component-derived SNOT-22 subdomain scores and general health-related quality of life.

\begin{tabular}{|c|c|c|c|c|}
\hline \multirow[b]{3}{*}{ Subdomain score } & \multicolumn{4}{|c|}{ EQ5D-VAS } \\
\hline & \multicolumn{2}{|c|}{ Univariate } & \multicolumn{2}{|c|}{ Multivariable } \\
\hline & $(95 \% \mathrm{Cl})$ & $P$ value & $(95 \% \mathrm{Cl})$ & $P$ value \\
\hline Sleep & $-6.20(-9.32--3.07)$ & $<0.001$ & $-5.80(-8.83--2.78)$ & $<0.001$ \\
\hline Nasal & $-3.50(-6.75--0.25)$ & 0.037 & $-4.06(-7.00--1.11)$ & 0.008 \\
\hline Otologic/facial pain & $-6.87(-9.96--3.78)$ & $<0.001$ & $-6.49(-9.47--3.51)$ & $<0.001$ \\
\hline \multirow[t]{3}{*}{ Emotional function } & $-3.86(-7.10--0.62)$ & 0.021 & $-3.38(-6.29--0.47)$ & 0.025 \\
\hline & \multicolumn{4}{|c|}{ HUV } \\
\hline & \multicolumn{2}{|c|}{ Univariate } & \multicolumn{2}{|c|}{ Multivariable } \\
\hline Sleep & $-0.04(-0.06--0.01)$ & 0.003 & $-0.03(-0.06--0.01)$ & 0.003 \\
\hline Nasal & $-0.02(-0.05-0.00)$ & 0.073 & $-0.03(-0.05-0.00)$ & 0.018 \\
\hline Otologic/facial pain & $-0.06(-0.08--0.04)$ & $<0.001$ & $-0.06(-0.08--0.03)$ & $<0.001$ \\
\hline Emotional function & $-0.04(-0.06--0.01)$ & 0.001 & $-0.04(-0.06--0.02)$ & $<0.001$ \\
\hline
\end{tabular}

${ }^{1}$ Controlling for age, gender, the presence of any aeroallergen hypersensitivity, comorbid asthma and nasal polyps. Abbreviations: EQ5D-VAS: EQ5D visual analog scale score, HUV: EQ5D-derived health utility value.

Table 2. Association between un-weighted SNOT-22 subdomain scores and general health-related quality of life.

\begin{tabular}{|c|c|c|c|c|}
\hline \multirow[b]{3}{*}{ Subdomain score } & \multicolumn{4}{|c|}{ EQ5D-VAS } \\
\hline & \multicolumn{2}{|c|}{ Univariate } & \multicolumn{2}{|c|}{ Multivariable } \\
\hline & $(95 \% \mathrm{Cl})$ & P value & $(95 \% \mathrm{Cl})$ & $P$ value \\
\hline Sleep & $-0.92(-1.18--0.65)$ & $<0.001$ & $-0.55(-0.96--0.13)$ & 0.011 \\
\hline Nasal & $-0.76(-1.07--0.44)$ & $<0.001$ & $-0.20(-0.59-0.19)$ & 0.317 \\
\hline Otologic/facial pain & $-1.91(-2.53--1.29)$ & $<0.001$ & $-0.76(-1.62-0.09)$ & 0.082 \\
\hline \multirow[t]{3}{*}{ Emotional function } & $-3.11(-4.57--1.64)$ & $<0.001$ & $-0.14(-1.87-1.60)$ & 0.878 \\
\hline & \multicolumn{4}{|c|}{ HUV } \\
\hline & \multicolumn{2}{|c|}{ Univariate } & \multicolumn{2}{|c|}{ Multivariable } \\
\hline Sleep & $-0.01(-0.01--0.00)$ & $<0.001$ & $-0.00(-0.01-0.00)$ & 0.116 \\
\hline Nasal & $-0.01(-0.01--0.00)$ & $<0.001$ & $0.00(-0.00-0.00)$ & 0.691 \\
\hline Otologic/facial pain & $-0.02(-0.02--0.01)$ & $<0.001$ & $-0.01(-0.02--0.00)$ & 0.004 \\
\hline Emotional function & $-0.03(-0.04--0.02)$ & $<0.001$ & $-0.01(-0.02-0.00)$ & 0.174 \\
\hline
\end{tabular}

${ }^{1}$ Controlling for age, gender, the presence of any aeroallergen hypersensitivity, comorbid asthma and nasal polyps Abbreviations: EQ5D-VAS: EQ5D visual analog scale score, HUV: EQ5D-derived health utility value.

effects on CRS severity. Sleep quality has been shown to be negatively impacted by CRS and this association is even greater in the setting of comorbid obstructive sleep apnea ${ }^{(26)}$. Cognitive dysfunction that arises as a result of distractibility or reduced concentration in the setting of CRS is associated with more severe CRS symptomatology ${ }^{(27)}$. More severe CRS symptomatology is also associated with higher reports of pain and increased risk of depression ${ }^{(28)}$ as well as decreased olfactory-specific $\mathrm{QOL}^{(29)}$. The severity of CRS-specific symptomatology may also be modified by patient characteristics. Factors such as aeroallergen hypersensitivity and comorbid asthma are associated with more severe CRS symptomatology $y^{(30,31)}$.

These previous disease-specific QOL studies have been critical to our understanding of how patients are affected by CRS-spe- 
cific symptom severity. However, while assessment of diseasespecific QOL is meant to maximize the sensitivity of detecting intra-individual disease-specific changes ${ }^{(32)}$, assessment of general health-related QOL allows for detection of differences between individuals at the general population level ${ }^{(15)}$. Additionally, general health-related QOL can be used to calculate health utility values associated with disease states, which may then be used to perform cost utility analyses of different treatment interventions. In fact, treatment of CRS leads to improvement in general health-related QOL. Recent economic analyses using general health-related QOL assessments also have shown the cost-effectiveness of CRS treatments such as ESS ${ }^{(19)}$. As the cost effectiveness of various CRS treatments is better defined, treatment algorithms will ideally identify those CRS patients who may stand to gain the greatest QOL improvement with different treatment options.

By identifying how certain symptoms relate to general healthrelated QOL, we may be able to maximize QOL gains with different CRS treatments that focus on alleviating specific symptoms. We and others have previously shown that CRS symptomatology may broadly be categorized as sleep-, nasal-, otologic/ facial pain-, and emotional function-related symptoms ${ }^{(21,22,33)}$. Due to the intuitive grouping of CRS symptomatology into these four categories, as well as previous work suggesting that these four symptom categories may represent different underlying pathophysiologic mechanisms of CRS, in this current study we sought to determine how these four symptom patterns impacted general health-related QOL in CRS patients. We specifically hypothesized that these categories of CRS symptoms may be differentially associated with the general health-related QOL impairment seen in CRS patients.

There are multiple methods for collectively quantifying the severity of similar CRS symptoms. Using the SNOT-22, calculation of CRS subdomain scores for specific categories of symptoms has been previously performed in two different ways. Because the notion of CRS symptom subdomains was derived using structural equation modeling methods such as factor analysis or PCA of the SNOT-22 $2^{(21,22,33)}$, PC-derived subdomain scores may be calculated as the sum of SNOT-22 item responses that are weighted by PC-derived weights (also referred to as loadings(22). For example, the sleep subdomain score could be calculated by summing the score of each SNOT-22 item that is weighted by the sleep PC loadings. Alternatively, a simpler approach to calculating subdomain scores is to assign specific SNOT-22 items to each subdomain and simply add the scores for those SNOT-22 items in an un-weighted manner ${ }^{(34)}$. The PCA-based method for deriving SNOT-22 subdomain scores benefits from accounting for the exact relationship of dominant symptoms with each other as well as by accounting for small contributions from all SNOT-22 items, rather than accounting for just the most dominant symptoms for each subdomain score. However, the
PCA-based method of calculating subdomain scores is analytically intensive and not amenable to convenient use in routine clinical practice. The alternative approach to accounting for only the dominant symptoms in each SNOT-22 subdomain and adding the scores of the corresponding SNOT-22 items in an unweighted manner is much simpler and convenient to use. This approach, however, does not account for the subtle mathematical relationships and correlations between the SNOT-22 item responses/scores.

In our study, we confirmed sleep, nasal, otologic/facial pain and emotional function symptomatology as distinct CRS disease patterns using a PCA. We then assessed these four symptom categories using both of the aforementioned methods for calculating SNOT-22 subdomain scores. We found that PC-derived SNOT-22 subdomain scores were significantly associated with EQ5D-based measures of general health-related QOL. Un-weighted SNOT-22 subdomain scores, however, were less sensitive for detecting association with EQ5D-based measures of general health-related QOL after accounting for other subdomain scores and other confounding variables. For both methods of calculating SNOT-22 subdomain scores, the otologic/facial pain subdomain demonstrated the greatest magnitude of association with our EQ5D-based measures of general health-related QOL. The sleep subdomain had the second largest effect on general health-related QOL and nasal symptoms had the smallest effect. Our findings with respect to PC-derived SNOT-22 subdomain scores further demonstrate that there likely exist four distinct pathophysiologic processes in CRS that result in distinct symptom patterns and that each of these pathophysiologic processes has a significant, but different, impact on CRS patients' general health-related QOL. However, further study is needed to confirm whether our findings are due to different pathophysiologic mechanisms of CRS.

Our findings may also have important implications for the consideration of different treatment strategies for patients with CRS. Targeting CRS symptoms that are most associated with a general health-related QOL impairment may lead to the greatest cost utility in the treatment of CRS. Further longitudinal study is needed to determine whether focusing interventions on the subdomains most dominant for patients will optimize gains in QOL. In the meantime, knowledge of the differential impact of CRS symptoms on patient QOL may allow a greater understanding of the overall effect of CRS on patients' lives.

\section{Acknowledgement}

The authors gratefully acknowledge the contribution of the patients who participated in this study.

\section{Authorship contribution}

LPH, KMP, RWB, DSC, STG performed the study and wrote/revised the manuscript. ARS designed, performed the study and 


\section{References}

1. Rosenfeld RM, Andes D, Bhattacharyya $\mathrm{N}$, et al. Clinical practice guideline: adult sinusitis. Otolaryngol Head Neck Surg 2007 Sep;137(3 Suppl):S1-31.

2. Fokkens WJ, Lund VJ, Mullol J, et al. European Position Paper on Rhinosinusitis and Nasal Polyps 2012. Rhinol Suppl 2012 Mar;(23)(23):3 p preceding table of contents, 1-298.

3. Van Crombruggen K, Zhang N, Gevaert P, Tomassen P, Bachert C. Pathogenesis of chronic rhinosinusitis: inflammation. J Allergy Clin Immunol 2011 Oct;128(4):728732.

4. Payne SC, Borish L, Steinke JW. Genetics and phenotyping in chronic sinusitis. J Allergy Clin Immunol 2011 Oct;128(4):710-20; quiz 721-2.

5. Sedaghat AR, Gray ST, Wilke CO, Caradonna DS. Risk factors for development of chronic rhinosinusitis in patients with allergic rhinitis. Int Forum Allergy Rhinol 2012 SepOct;2(5):370-375.

6. Gudis D, Zhao KQ, Cohen NA. Acquired cilia dysfunction in chronic rhinosinusitis. Am J Rhinol Allergy 2012 Jan-Feb;26(1):1-6.

7. Sedaghat AR, Phipatanakul W, Cunningham MJ. Atopy and the Development of Chronic Rhinosinusitis in Children with Allergic Rhinitis. J Allergy Clin Immunol Pract 2013;1(6):689-691.

8. Sedaghat AR, Gray ST, Chambers KJ, Wilke CO, Caradonna DS. Sinonasal anatomic variants and asthma are associated with faster development of chronic rhinosinusitis in patients with allergic rhinitis. Int Forum Allergy Rhinol 2013 Sep;3(9):755-761.

9. London NR, Lane AP. Innate Immunity and Chronic Rhinosinusitis: What We Have Learned From Animal Models. Laryngoscope Invest Otolaryngol 2016;1(3):49-56.

10. Sedaghat AR, Bhattacharyya N. Chronic rhinosinusitis symptoms and computed tomography staging: improved correlation by incorporating radiographic density. Int Forum Allergy Rhinol 2012 SepOct;2(5):386-391.

11. Ryan WR, Ramachandra T, Hwang PH. Correlations between symptoms, nasal endoscopy, and in-office computed tomography in post-surgical chronic rhinosinusitis patients. Laryngoscope 2011 Mar;121(3):674-678.

12. DeConde AS, Soler ZM. Chronic rhinosinusitis: Epidemiology and burden of disease. Am J Rhinol Allergy 2016 Mar;30(2):134-139.

13. Caulley L, Thavorn K, Rudmik L, Cameron C, Kilty SJ. Direct costs of adult chronic rhinosinusitis by using 4 methods of estimation: Results of the US Medical Expenditure Panel Survey. J Allergy Clin Immunol 2015
Dec;136(6):1517-1522.

14. Rudmik L, Hopkins C, Peters A, Smith TL, Schlosser RJ, Soler ZM. Patient-reported outcome measures for adult chronic rhinosinusitis: A systematic review and quality assessment. J Allergy Clin Immunol 2015 Dec;136(6):1532-40.e1-2.

15. EuroQol Group. EuroQol--a new facility for the measurement of health-related quality of life. Health Policy 1990 Dec;16(3):199-208.

16. Remenschneider AK, D'Amico L, Gray ST, Holbrook EH, Gliklich RE, Metson R. The EQ-5D: a new tool for studying clinical outcomes in chronic rhinosinusitis. Laryngoscope 2015 Jan;125(1):7-15.

17. Luk LJ, Steele TO, Mace JC, Soler ZM, Rudmik L, Smith TL. Health utility outcomes in patients undergoing medical management for chronic rhinosinusitis: a prospective multiinstitutional study. Int Forum Allergy Rhinol 2015 Nov;5(11):1018-1027.

18. Rudmik L, Smith TL, Mace JC, Schlosser RJ, Hwang PH, Soler ZM. Productivity costs decrease after endoscopic sinus surgery for refractory chronic rhinosinusitis. Laryngoscope 2016 Mar;126(3):570-574.

19. Scangas GA, Su Bs BM, Remenschneider AK, Shrime MG, Metson R. Cost utility analysis of endoscopic sinus surgery for chronic rhinosinusitis. Int Forum Allergy Rhinol 2016 Mar 17.

20. Olowosusi OZ, Asoegwu CN, Olagunju AT, Nwawolo CC. A cross-sectional evaluation of the correlation between disease severity and quality of life in chronic rhinosinusitis patients in Nigeria. Eur Arch Otorhinolaryngol 2015 Sep;272(9):23412346.

21. DeConde AS, Bodner TE, Mace JC, Smith TL. Response shift in quality of life after endoscopic sinus surgery for chronic rhinosinusitis. JAMA Otolaryngol Head Neck Surg 2014 Aug;140(8):712-719.

22. Sedaghat AR, Gray ST, Caradonna SD, Caradonna DS. Clustering of chronic rhinosinusitis symptomatology reveals novel associations with objective clinical and demographic characteristics. Am J Rhinol Allergy 2015 Mar-Apr;29(2):100-105.

23. Hopkins C, Gillett S, Slack R, Lund VJ, Browne JP. Psychometric validity of the 22-item Sinonasal Outcome Test. Clin Otolaryngol 2009 Oct;34(5):447-454.

24. Cohen J. Statistical power analysis for the behavioral sciences. Hillsdale NJ: L. Erlbaum Associates; 1988.

25. Ference EH, Stubbs V, Lidder AK, et al. Measurement and comparison of health utility assessments in chronic rhinosinusitis. Int Forum Allergy Rhinol 2015 Oct:5(10):929-936.

26. Alt JA, DeConde AS, Mace JC, Steele TO, Orlandi RR, Smith TL. Quality of Life in
Patients With Chronic Rhinosinusitis and Sleep Dysfunction Undergoing Endoscopic Sinus Surgery: A Pilot Investigation of Comorbid Obstructive Sleep Apnea. JAMA Otolaryngol Head Neck Surg 2015 Oct;141(10):873-881.

27. Tarasidis GS, DeConde AS, Mace JC, et al. Cognitive dysfunction associated with pain and quality of life in chronic rhinosinusitis. Int Forum Allergy Rhinol 2015 Nov;5(11):1004-1009.

28. Cox DR, Ashby S, DeConde AS, et al. Dyad of pain and depression in chronic rhinosinusitis. Int Forum Allergy Rhinol 2016 Mar;6(3):308-314.

29. Soler ZM, Smith TL, Alt JA, Ramakrishnan VR, Mace JC, Schlosser RJ. Olfactory-specific quality of life outcomes after endoscopic sinus surgery. Int Forum Allergy Rhinol 2016 Apr;6(4):407-413.

30. Davila I, Rondon C, Navarro A, et al. Aeroallergen sensitization influences quality of life and comorbidities in patients with nasal polyposis. Am J Rhinol Allergy 2012 Sep-Oct;26(5):e126-31.

31. Rix I, Hakansson K, Larsen CG, Frendo M, von Buchwald C. Management of chronic rhinosinusitis with nasal polyps and coexisting asthma: A systematic review. Am J Rhinol Allergy 2015 May-Jun;29(3):193-201.

32. Piccirillo JF, Merritt MG,Jr, Richards ML. Psychometric and clinimetric validity of the 20-Item Sino-Nasal Outcome Test (SNOT20). Otolaryngol Head Neck Surg 2002 Jan;126(1):41-47.

33. Pynnonen MA, Kim HM, Terrell JE. Validation of the Sino-Nasal Outcome Test 20 (SNOT20) domains in nonsurgical patients. Am J Rhinol Allergy 2009 Jan-Feb;23(1):40-45.

34. DeConde AS, Mace JC, Alt JA, Rudmik L, Soler ZM, Smith TL. Longitudinal improvement and stability of the SNOT-22 survey in the evaluation of surgical management for chronic rhinosinusitis. Int Forum Allergy Rhinol 2015 Mar;5(3):233-239.

Ahmad R. Sedaghat, MD, PhD

Department of Otolaryngology Massachusetts Eye and Ear Infirmary 243 Charles Street Boston, MA USA 02114

Tel: +001 617-573-6011

Fax: +001 617-573-6845

E-mail:

ahmad_sedaghat@meei.harvard.edu 